\title{
ORIENTATION CHARACTERISTICS OF THE MICROSTRUCTURE
}

J.Pospiech ${ }^{*}$, K.Lucke ${ }^{* *}$ and K.Sztwiertnia ${ }^{*}$

* Instytut Podstaw Metalurgii PAN, Krakow, Poland

Institut fur Allgemeine Metallkunde und Metallphysik der RWTH, Aachen, Germany

\begin{abstract}
A systematization of orientation characteristics describing the various orientation aspects of the microstructure of the polycrystalline material is presented.The statistical quantities which are functions of orientation or orientation differences are defined on the set of orientation measurements or derived from the conditional orientation correlation function (presented by Adams). The variety of the orientation characteristics comprises principal distributions which are texture functions and such which are partial distribution for the description of correlation between orientation. The presented analysis is based mainly on the use of regular grids for selecting the measuring points of orientations. The usefulness is demonstrated by an example.
\end{abstract}

\section{INTRODUCTION}

The properties and anisotropy of a polycrystalline material are strongly related to its microstructural characteristics. Such statistical quantities which are defined on the spatial arrangements of different phases and grains and their crystallographic orientations expose the preferences and the the correlations occurring in the microstructure.These quantities are in general functions of many parameters but (depending on the problem under investigation and on the material and its state) the most required information is supplied by various particular forms of the characteristics with a small number of parameters (see e.g. H.J.Bunge [1]).

Essential forms of characteristics are defined on arrangements of the orientation measuring points which can be picked with reference to certain spatial elements of the microstructure such as grains, grain boundaries, various kinds of deformation inhomogenities etc. In other cases in which,for example, details of these spatial elements are not sufficiently distinguishable as it is often the case in deformed samples, they can be arranged in the form of random arrays or of regular grids. The arrangement of the orientation measuring points which must be statistically homogeneous represents the basic source of information about the orientation aspects of the microstructure.

If the geometry of locations of the measuring points is established, then this situation can be described by the conditional form of the two-point orientation correlation function (COCF) $\mathrm{C}\left(\mathrm{g}^{\prime}, \mathrm{g}^{\prime \prime} / \mathrm{r}\right)$ described and discussed by B.Adams [2]. According to its definition $d N / N:=C\left(g^{\prime}, g^{\prime \prime} \mid r\right) d g^{\prime} d g^{\prime \prime}$ the COCF gives the frequency $\mathrm{dN} / \mathrm{N}$ of the occurance of orientations g' and $g$ " from the interval $\mathrm{dg}$ ' and $\mathrm{dg}$ ', respectively, when their measuring points are separated by a fixed vector $r$. In particular cases a many-point correlation function may be preferred.

In general, in the analysis of the various orientation aspects of the microstructure one-point forms of distributions which can be represented in the 3-dimentional orientation space are of special interest.In a earlier paper by A.Morawiec and J.Pospiech [3] some basic relation concerning the COCF and some one-point forms of the microstructural quantities have been derived and discussed.In the present paper basing on the results in $[3,4]$ these treatments have been extended in view of direct applications by syste- 
matizing the distribution functions named here orientation characteristics. An example of application will be demonstrated using a set of orientation measurements arranged in a form of a regular grid for rolled copper supplied by Long [5].

\section{ORIENTATION CHARACTERISTICS}

There exist various kinds of orientation characteristics which are chosen depending on the nature of the considered problems. They can be derived from the many-point COCF or directly defined on the set of measured orientations. A general systematization of the orientation characteristics is given in Table 1.

All these functions represent distributions of orientations or distributions of orientation differences. This means that both types of function are described in the orientation space. Among these functions the texture functions are distinguished as the principal distribution. There are two kinds of texture functions which are distribution densities formed on a whole set of measurements independently of the locations of the measuring points in the sample space.

The first kind formed on a set of orientations is the well known Orientation Distribution Function (ODF ) $f(g)$ [5] - the most important characteristics of the microstructure. It is defined by the expression $\Delta N(g) / N:=f(g) \Delta g$, where $N$ is the total number of the measuring points in the sample space and $\Delta \mathrm{N}(\mathrm{g}) / \mathrm{N}$ is the frequency of occurrence of an orientation in the range $\Delta \mathrm{g}$ around $\mathrm{g}$.

The second one is completely determined by the texture and is created on the set of orientation differences $\Gamma=g^{\prime} g^{\prime \prime-1}$ between the orientations $g^{\prime}$ and g" for any two measuring points in the sample. This is the Orientation Difference Distribution Function (ODDF, $u(\Gamma)$ defined by $\Delta \nu(\Gamma) / \nu:=u(\Gamma) \Delta \Gamma$ where $D$ is the total number of pairs of the orientation measuring points and $\Delta \nu(\Gamma) / \nu$ is the frequency of the occurrence of the orientation difference inside the range $\Delta \Gamma$ around $\Gamma$.

As can be seen from the scheme (Table 1, Eq(2) or Eq(3)) these texture functions represent the particular case of one-point forms of distribution densities obtained by integration of the two-point COCF.

For a detailed study of local regularities which may exist between orientations in the polycrystalline matrix partial distributions are introduced. According to the scheme in Table 1 there are partial distribution functions of orientations (partial ODF's $H(g)$ ) and such of orientation differences (partial ODDF's V $(\Gamma)$ ). Measurements used for the various partial distribution functions are selected from the total sets by certain conditions determined depending on the problem under consideration. Some of these functions can be analytically connected with the two-point COCF.

Partial distributions are here expressed by using the weight factors Qis1 or $\mathrm{T} j \leq 1$ assigned to the orientation measuring points $\mathrm{Pi}_{i}$ or to each pair $\mathrm{pj}_{\mathrm{j}}$ of orientation measuring points (when orientation differences are considered), respectively. This includes the cases when the weight factors assume the values 1 or 0 or when they can vary between 1 and 0 , respectively. The partial distributions are defined (and normalized to one) similarly as the principal distributions when the corresponding weight factors $Q i$ or $T j$ are taken into account.

The partial ODF $H(g)$ is then defined by the expression

$$
\mathrm{H}(\mathrm{g}) \Delta \mathrm{g}:=-\frac{\Delta Q}{Q}(\underline{\mathrm{g}})-=-\frac{\Delta Q}{\Delta} \frac{(\mathrm{g})}{\Delta}(\mathrm{g})-\cdot-\frac{\Delta N}{N}(\mathrm{~g}) \cdot-\frac{N}{Q}-
$$

where $Q$ is the sum of the weight factors $Q i$ counted from the whole set of $N$ orientation measuring points, and $\Delta Q(g)$ is the sum of weight factors belonging to the $\Delta \mathrm{N}(\mathrm{g})$ measuring points whose orientations fall into the 
range $\Delta g$ around $g$. Expression (1) leads to the form (4) and to the texture reduced form (6) in Table 1 , where $K(g)=(\Delta Q(g) / \Delta N(g)) / \bar{Q}$ is the statistical weight which in the orientation space occur for a given

TABLE 1

GENERAL SYSTEMATIZATION OF THE ORIENTATION CHARACTERISTICS and some relations obtained from the COCF

Conditional two-point orientation correlation function (COCF)

$$
\mathrm{C}\left(\mathrm{g}, \mathrm{g}^{\prime} \mid \mathbf{r}\right)
$$

Integration over the volume $\mathbf{v}$ in a statistically homogeneous sample space

$$
-\frac{1}{V}-\int_{v} C\left(g, g^{\prime} \mid r\right) d_{3} r=f(g) f\left(g^{\prime}\right)
$$

For random spatial arrangement: $C_{\text {rand }}\left(g^{\prime}, g^{\prime}\right)=f(g) f\left(g^{\prime}\right)$

Texture reduced COCF: $c\left(g_{,}, g^{\prime} \mid r\right):=C\left(g, g^{\prime} \mid r\right) / f(g) f\left(g^{\prime}\right)$

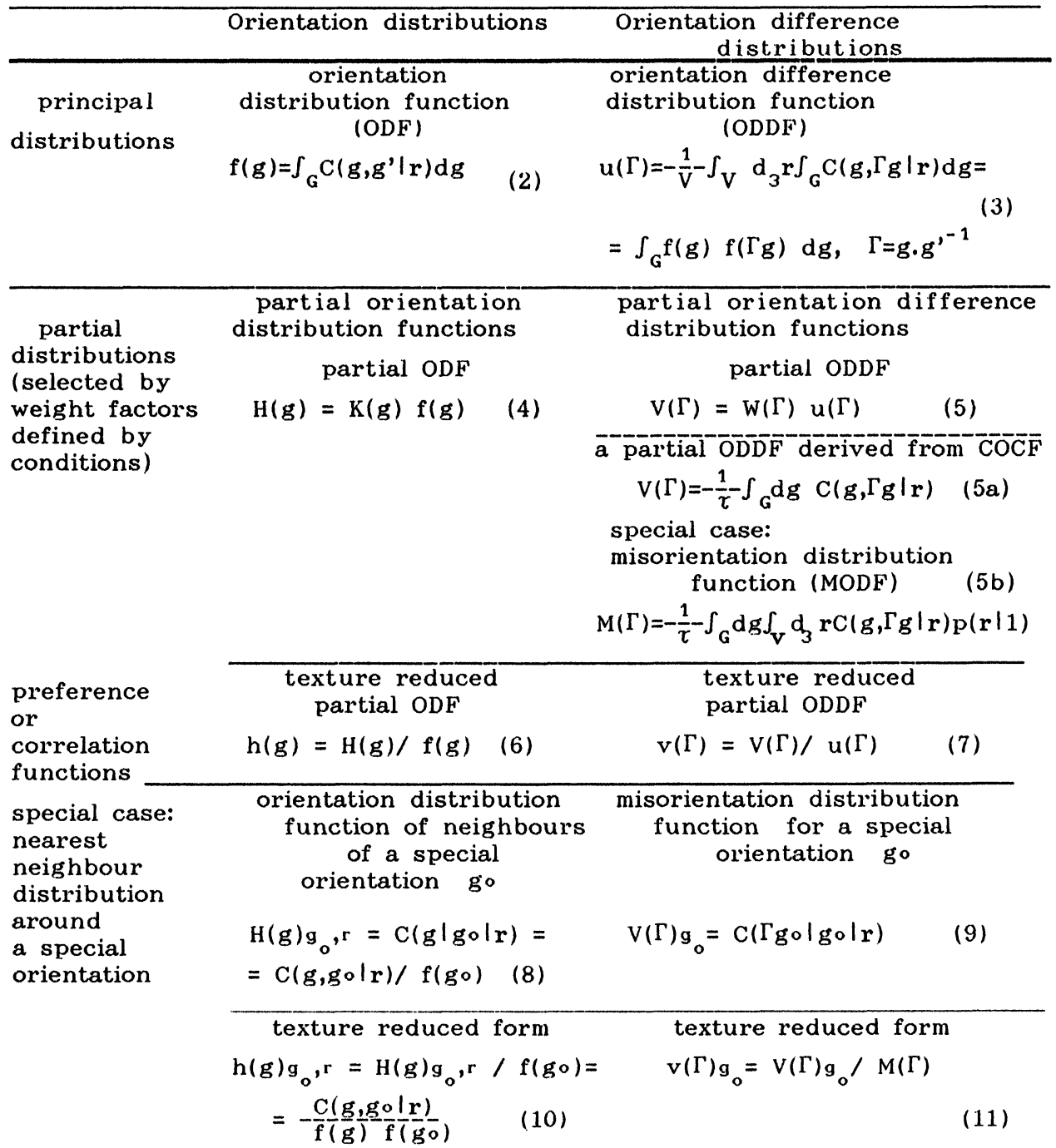


orientation referred to $\bar{Q}=Q / N$, i.e. to the weight factor which on the average occurs for one measuring point.

Analogously, the partial ODDF $V(\Gamma)$ is defined when the weight factors $T j$ selecting the orientation differences are used. The corresponding relation following from the definition is given in Table 1 by Eq(5) and the texture reduced form by $\mathrm{Eq}(7)$.

The texture reduced partial distributions $h(g)=K(g)$ or $v(\Gamma)=\tau(\Gamma)$ (Eq (6) and $\mathrm{Eq}(7)$ ) will be denoted as "preference functions". They express how much more frequently an orientation or orientation difference,respectively,occurs if the weight factors are considered in comparison with the cases when the weight factors are not considered.

Among the partial ODF very important is the special case $\mathrm{H}(\mathrm{g})$ go or $\mathrm{h}(\mathrm{g}) \mathrm{go}$ formed by those orientations whose measuring points $P^{\prime}$ are seperated from the locations $P_{0}$ of a special orientation go by the vector $r$ (or by the

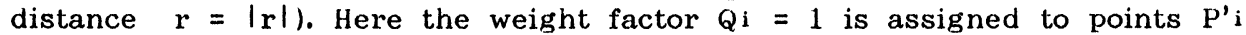
and the factor $Q_{i}=0$ is assigned to the others.According to Eq(8) and Eq(10) in Table 1 these functions are directly connected with the two-point COCF. For a sufficiently large distance $r$ from $P_{0} \quad(r \geq r c)$ the correlations disappear and one obtains $H(g) g o \approx f(g)$. The other limit case is when the points with $Q i=1$ are chosen to be the nearest neighbour points to go.

The first and essential form of the partial ODDF $V(\Gamma)$ is obtained by selecting pairs of such measuring points which are seperated by the fixed vector $\mathbf{r}$ (or by the distance $\mathbf{r}$ ). To orientation differences of such pairs the weight factor 1 is ascribed,to others- the weight factor 0 . This form of describing correlations between orientations is connected with the COCF by $\mathrm{Eq}(5 \mathrm{a})$ shown in Table 1.

If the distance $r$ is sufficiently large $(r \geq r c)$, then the correlation disappears leading to $T(\Gamma)=1$ and $V(\Gamma) \approx u(\Gamma)$. Of great importance is the other limit case in which $\mathbf{r}$ is the nearest neighbour distance. The resulting partial ODDF, the misorientation distribution function (MODF) $V(\Gamma) \equiv M(\Gamma)$ describes the distribution density of orientation differences $\Gamma$ to the nearest neighbour measuring points. A general form of the MODF is given by $\mathrm{Eq}(5 \mathrm{~b})$ in Table 1 , where $\mathrm{p}(\mathrm{r} / 1)$ denotes the probability density that two points of neighbouring crystallites are separeted by the vector $r$.

A further kind of a partial $O D D F$ is defined on a subset of orientation differences $\Gamma$ selected by additional conditions. An important example is based on the additional condition that the pairs of orientations separated by the fixed vector $r$ contain the special orientation go. The resulting partial ODDF $\mathrm{V}(\Gamma)$ go follows from the conditional form of the $\mathrm{COCF}$ expressed by Eq(9) in Table 1. In order to obtain the preference function $v(\Gamma)$ go it appears here reasonable to relate $v(\Gamma)$ go not to the ODDF $u(\Gamma)$ but to the MODF $M(\Gamma)(E q(11)$ Table 1).

\section{AN EXAMPLE OF APPLICATIONS}

The presented method described by a simple system of orientation characteristics is easily applicable in the analysis of the orientation aspects of the microstructure. The usefulness of such analysis was demonstrated in [4] on examples based on a set of orientation measurements arranged in form of a regular (quadratic) grid of a copper sample rolled to $97 \%$ reduction supplied by Long [5]. The number of measured orientations was 165, i.e. relatively small, so that the theoretical possibilities of the analysis could not be fully utilized. In the present paper using the same data set only one example will be shown to illustrate the method of analysis. Fourier series up to the range 34 were used in the calculations.

In general, the basic initial information which is necessary for a detailed analysis is supplied by the ODF and by the MODF. Here some results obtained basing on the MODF $m(\Gamma)$ will be analysed. This partial ODDF characterizes 
the distribution of the orientation differences $\Gamma_{j}$ of pairs of the nearest orientation measuring points. These pairs obtain the weight factor $T \mathrm{j}=1$, and all others- $\mathrm{T} j=0$. In each case 8 (not 4 ) nearest neighbour points were used and the number of orientation differences was equal to 737 .

In fig. 1 the MODF $m(\Gamma)=M(\Gamma) / u(\Gamma)$ is plotted in the space of rotation axes $a$ and rotation angles $\omega$ on the sections $\omega=$ const in the region of disorientations. The obtained MODF $m(\Gamma)$ reveals some correlations characterized by a maximum in $\langle 111\rangle 50^{\circ}$ and by a weaker one in $\langle 112\rangle 55^{\circ}$ and in some others.
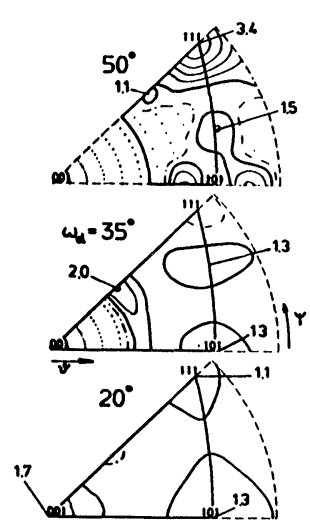
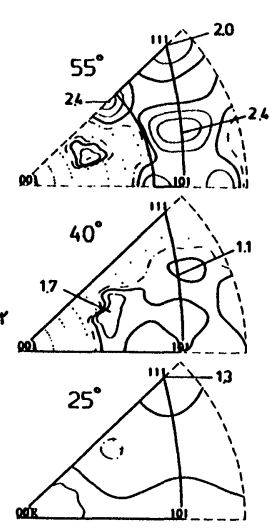
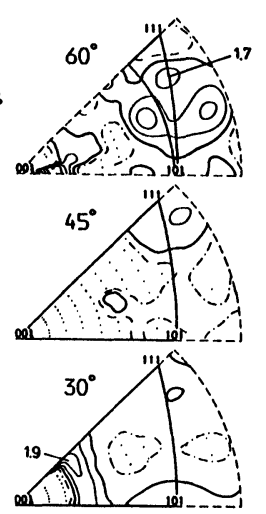

Fig.1

The misorientation distribution function $m(\Gamma)=M(\Gamma) / u(\Gamma)$ (normalized by the ODDF)

For a closer examination of the observed correlations the probability will be investigated that a given orientation is connected with its nearest neighbour orientation by $\langle 111\rangle 50^{\circ}$ or $\langle 112\rangle 55^{\circ}$ rotations. For this reason the weight factors $Q^{11}=N i(<111>50) / 8$ or $Q^{112}=N i(<112>55) / 8$ are ascribed to each measuring point $\mathrm{P}_{i}$ being equal to the relative number of neighbours for which the corresponding rotation angles $(\omega=50$ or $\omega=55)$ lie within the range $\omega-5 \leq \omega \leq \omega+5$ and the corresponding rotation axes $(a=\langle 111\rangle$ or $a=\langle 112\rangle$ ) deviate from the exact positions by less than $15^{\circ}$.

Fig.2a shows the preference function $\mathrm{h}^{1}(\mathrm{~g})$ selected by weights $\mathrm{Q}^{111}$ presented in the space of Euler angles. This function reveals two lines of orientations which are distinguished by a high number of nearest neighbours forming the relationship of $\left\langle 111>50^{\circ}\right.$ to the orientations g. Both pass the main rolling component in the position $C=(112)[\overline{1} 11]$ and $S=(213)[\overline{3} \overline{6} 4]$. The one of the line indicated by $X$ shows the maximum values of $\approx 4.0$ in the range $60^{\circ}<\phi 2<<75^{\circ}$ and the other one,indicated by $Y$, the highest values of $\approx 8.0$ within the range $40^{\circ}<\phi 2<55^{\circ}$. Thus in the above ranges the orientations of the $\mathrm{X}$-line show a preference of 4 times and those of $\mathrm{Y}$-line a preference of 8 times for the occurrence of the relation $<111>50^{\circ}$ compared with the average frequency $\bar{Q}=0.073$,i.e. $1 / 2$ neighbours of each measuring point. It could be also shown that if one orientation of the pair forming the relation $<111>50^{\circ}$ is lying on $X$, then the other one is mostly found in $Y$, and vice versa. For example, the partners of the orientations forming the maximum $Y 1$ are mostly scattered in the range $\mathrm{X}_{1}-\mathrm{X}_{2}-\mathrm{X} 3$. Also the $\mathrm{B}$-orientation with the preference of 1.9 have the relationship $<111>50^{\circ}$, the positions of such neighbours are indicated by $\beta$.

Fig.2b shows the preference function $h^{11}(\mathrm{~g})$ selected by the weight $Q i$ exposing orientations related with the nearest neighbours in the sample space by the rotation $\left\langle 112>55^{\circ}\right.$. The highest concentration of $h^{1}\{\mathrm{~g})$ run over the main rolling components from C-position $\left(\phi 2=55^{\circ}\right)$ to $B$-position pass the $\mathrm{S}$-component splitting in its neighbourhood $\left(\$ 2=65^{\circ}\right)$ into three regions. The concentrations are more spread and their highest values are much lower than 
in the former case and do not exceed 2.5. The values show preferences referred to the unit of $\bar{Q}=0.05$ which corresponds to about $1 / 3$ neighbours on the average for each measuring point.

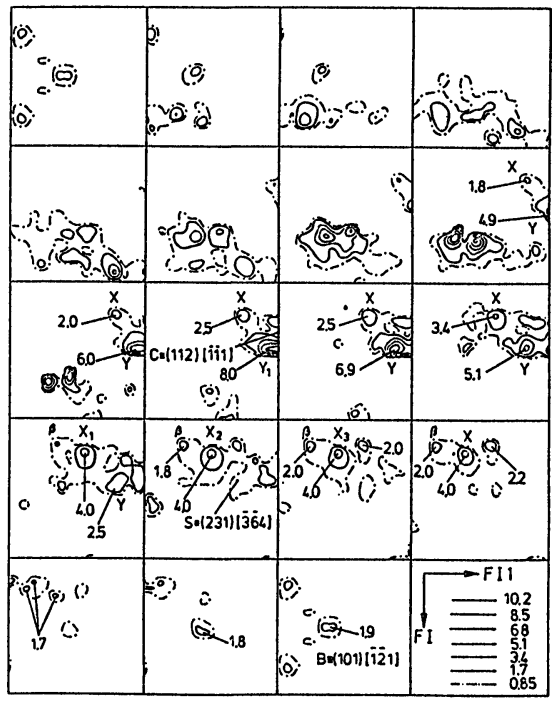

Fig.2a. The $\mathrm{h}^{11} \mathrm{~g}$ ) function

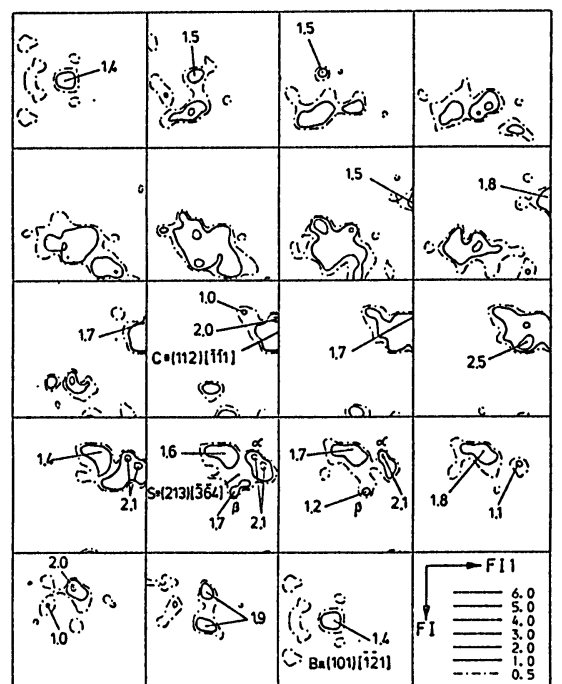

Fig.2b. The $\mathrm{h}^{11} q_{\mathrm{g}}$ ) function The normalized (texture reduced) partial ODFs

(a) revealing the $\left\langle 111>50^{\circ}\right.$-relation

(b) revealing the $\langle 112\rangle 55$ - relation

Similarly as before no preferences are recognized for the S-orientations and for the B-orientations with the maximum of 1.4 neighbours creating the relation $\left\langle 112>55^{\circ}\right.$ were found in the positions indicated by $\beta$. In the present case (in oposite to the former) the C-orientations are preferred by a maximum of 2.0 and the recognized neighbours creating the above relation are indicated by $\alpha$.

The obtained results characterised by distinguished relations of the type $<111>50^{\circ}$ and $\left\langle 112>55^{\circ}\right.$ are created by positions which are not typical for the most observed ODFs of rolled copper.It is possible that it has been measured accidentally in a special region of inhomogenities.

\section{CONCLUSIONS}

The orientation characteristics the general systematization of which is given in Table 1 provides basic information on microstructure by allowing identification and description of occurring preferences and correlations. Using partial distributions with appropriately chosen weight factors these microstructural regularities can be exposed and analysed.

\section{REFERENCES}

1.H.J.Bunge, Texture Analysis in Material Science (Butterworts, London 1982)

2.B.L.Adams,T.T.Wang and P.R.Morris, Proc.ICOTOM8,(SantaFe 1987), p.135 3.A.Morawiec and J.Pospiech, submitted to Texture and Microstructure, Feb.1990

4.J.Pospiech,K.Lucke and K.Sztwiertnia, to be published

5.N.J.Long,M.H.Loretto and R.E.Smallman, (Eurem 80, The Hague, 1980) 